\title{
Validation of a Novel Smartphone Accelerometer-Based Knee Goniometer
}

\author{
Matthew Ockendon, M.B.B.S., B.Sc., M.R.C.S. ${ }^{1}$ \\ Robin E. Gilbert, M.B.B.S., B.Med.Sci., F.R.C.S. (Tr \& Orth), Dip. (SEM) ${ }^{1}$ \\ ${ }^{1}$ Department of Sports Surgery, Robert Jones and Agnes Hunt \\ Orthopaedic Hospital, Gobowen, Oswestry, United Kingdom \\ J Knee Surg 2012;25:341-346.

\begin{abstract}
Address for correspondence and reprint requests Robin Gilbert, M.B.B.S., B.Med.Sci., F.R.C.S., Dip. (SEM), Department of Sports Surgery, Robert Jones and Agnes Hunt Orthopaedic Hospital, Gobowen, Oswestry SY10 7AG, United Kingdom
\end{abstract} \\ (e-mail: drrobgilbert@live.co.uk).
}

\begin{abstract}
Keywords

- fixed flexion

- knee

- goniometer

- accelerometer
\end{abstract}

Loss of full knee extension following anterior cruciate ligament (ACL) surgery has been shown to impair knee function. $^{1,2}$ However, there can be significant difficulties in accurately and reproducibly measuring a fixed flexion of the knee. Some studies suggest that conventional lower limb goniometry may contain errors of between 5 and 10 degrees even in experienced hands. ${ }^{3-5}$ Such errors may exceed the magnitude of a given fixed flexion deformity.

We sought to find a means to eliminate the difficulties associated with palpating the boney landmarks of the femurby making calculations based entirely on the measured position of the tibia. In the setting of state funded healthcare, even a relatively small capital outlay can be prohibitive-we therefore repurposed commercially available technology.

We studied the interobserver and the intraobserver reliabilities of our novel, accelerometer based, knee goniometer technique and compared it with a long-armed conventional goniometer for the assessment of fixed flexion knee deformity. Measurements on radiographs would provide a robust "gold standard" but the use of X-ray for this purpose is not routine and not without risk. We therefore chose the best commonly applied "bed-side" measurement technique. received

August 9, 2011

accepted after revision

October 5, 2011

published online

May 3, 2012
Copyright $\odot 2012$ by Thieme Medical Publishers, Inc., 333 Seventh Avenue, New York, NY 10001, USA. Tel: +1(212) 584-4662.
DOI http://dx.doi.org/ 10.1055/s-0031-1299669. ISSN 1538-8506. 
Accelerometers have been used to make anatomical measurements including measurements of knee flexion. ${ }^{6}$ These research studies demonstrated encouraging reproducibility and validity with useful "smallest detectable differences" (SDDs). Until relatively recently, however, these were the domain of research science-expensive, not widely available and often requiring additional computer hardware to make meaningful interpretations of the data obtained.

Increasingly, so-called "smartphones" use accelerometers. They serve as position sensors and as a means to interact with computer games. These hand-held devices present a powerful potential tool-not only do they contain high-quality triaxial accelerometers but also significant computing power, storage space, and internet connectivity.

\section{Materials and Methods}

\section{Accelerometer/Trigonometric Estimation of Knee Flexion}

A digital accelerometer returns a value in direct proportion to the acceleration it experiences. At rest it will return a value equal to the acceleration of free-fall $(1 \mathrm{G})$ when orientated perpendicular to the ground and zero acceleration when parallel to it.

By arranging three accelerometers in the $\mathrm{X}, \mathrm{Y}$, and $\mathrm{Z}$ axes it is possible to establish the orientation of a device by simple trigonometry.

The technique described here could be applied to any programmable device containing accelerometers. We used an iPhone ${ }^{\circledR}$ 3GS (Apple Inc., Cupertino, CA) for this study but numerous suitable smartphones are available on the market. Software applications do exist which use the built-in accelerometers to measure the inclination of the device and thereby measure angles. We took this a step further by writing a custom application to make calculations in real time according to the following model.

We may consider the femur, knee, and tibia to form two right-angled triangles (-Fig. 1). From the work of Anderson, ${ }^{7}$ we know that in the phenotypically normal adult the femur and tibia have a fairly constant relationship of length. Average ratio for men and women is $\sim 1.2$. Small differences in this ratio have a modest effect on the calculated angle (a change of $10 \%$ leads to less than a $5 \%$ change in the calculated angle).

An accelerometer-based inclinometer can easily be placed along the subcutaneous border of the tibia to measure its inclination relative to the ground. The femoral segment, by virtue of its anatomy, is significantly more difficult to instrument in this fashion. By accepting a constant ratio of lengths femur:tibia, it is possible to calculate the knee flexion angle (K) from the tibial inclination $(\beta)$ alone ( $\mathbf{F i g . ~ 1 ) ~ t h u s ~ e l i m i - ~}$ nating the need to measure the femoral position. This calculation is performed in real time by the smartphonereturning a value for knee flexion (or extension) angle based on the calculated position of the femur.

This technique requires the patient to be supine. Differences in body habitus between patients may have an impact on the measured inclination which is compensated for by using the contralateral limb as the control.

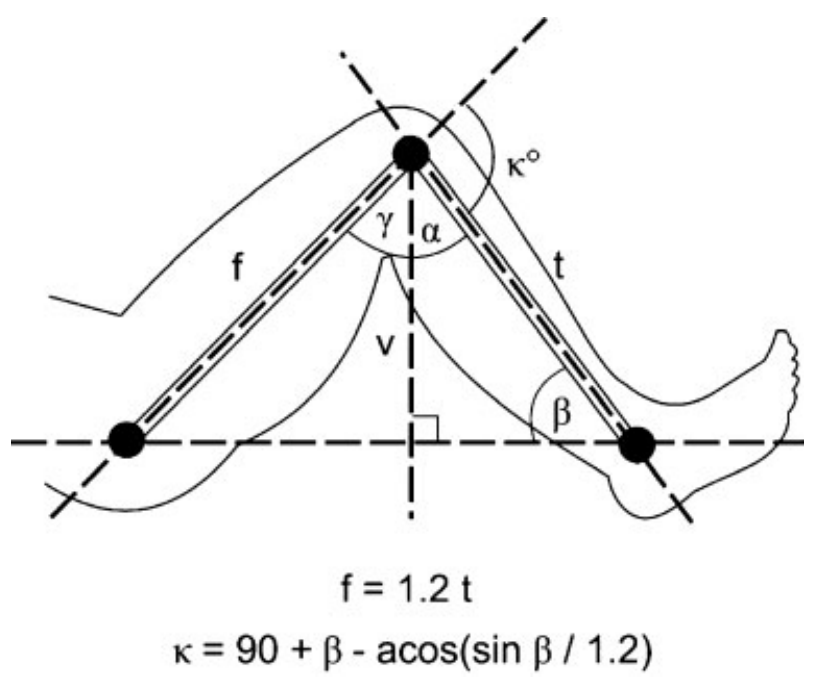

Figure 1 Calculation of knee flexion angle by trigonometry from measured tibial inclination.

Using this technique knee flexion angles between 145 degrees hyperextension and 145 degrees flexion can be measured-encompassing all but the slimmest and most flexible of knees.

With both measurement techniques, we took the "normal" knee as our zero point and then calculated the flexion/ extension of the "affected" knee relative to it.

\section{Data Collection}

Five healthy male volunteers (age range 30 to 40 years) were studied. None had known pre-existing knee pathology. Heights ranged from 1.74 to $1.86 \mathrm{~m}$. Weights ranged from 78 to $101 \mathrm{~kg}$. A bolster was used to symmetrically raise the heels off the examination couch. In this position, the soft tissues of the calf and thigh were clear of the couch and the knees fully extended. A series of fixed flexion deformities of between 5 and 45 degrees were simulated in each subject's right knee by the use of a series padded, solid, wedges placed behind the popliteal fossa with the subject supine ( $\mathbf{- F i g . 2 A}$ ).

Measurements of knee flexion angle were made with a telescopic-armed goniometer (Lafayette Instrument, Lafayette, IN) the instrument was centered on the lateral femoral epicondyle and the center points of the greater trochanter and lateral malleolus were palpated (-Fig. 2B). The same knee positions were also measured using the smartphone (iPhone 3GS, Apple Inc., CA) knee goniometer running the application "Knee Goniometer"8 (-Fig. 2C).

Each subject had three different knee flexion angles simulated. Two experienced, independent observers measured each position twice, separated by a time interval, with each instrument. The observers were blinded to the previous measurements. In total 120 measurement pairs (comparison of "normal" and "affected" sides) were recorded-30 different knee positions in all.

\section{Statistical Analysis}

Statistical analysis was undertaken in R (version 2.11.0-The $\mathrm{R}$ Foundation for Statistical Computing, Vienna, Austria). 

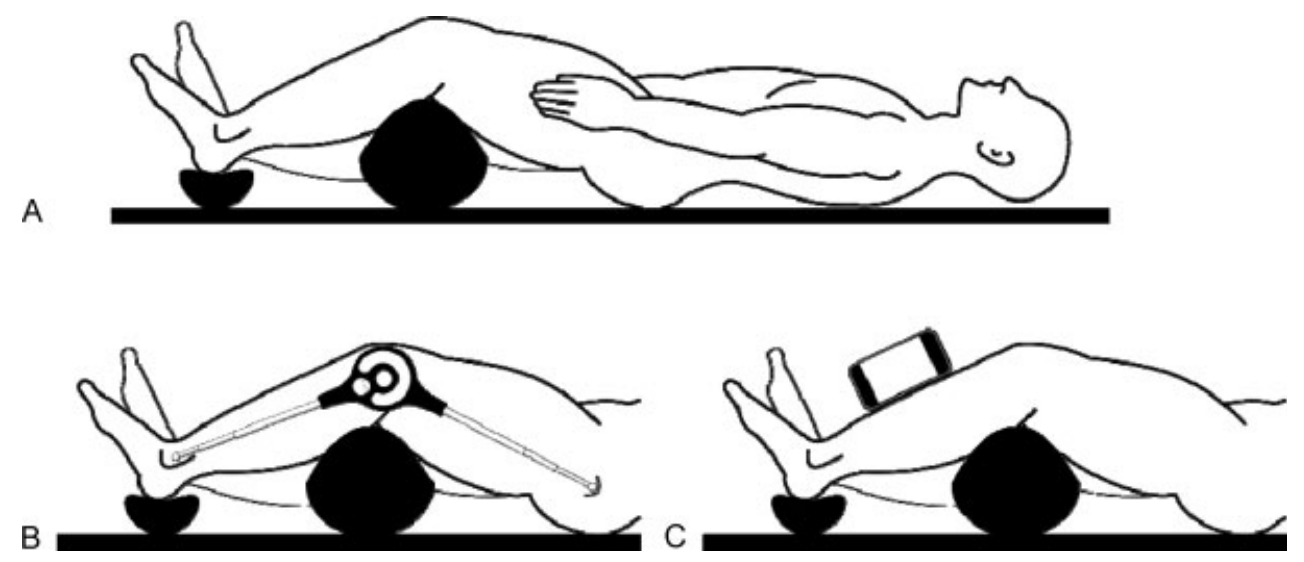

Figure 2 Patient setup and measurement techniques. (A) Bolster positioned behind popliteal fossa to stimulate a fixed flexion deformity. (B) Measurement with the Lafayette goniometer. (C) Measurment with the iPhone goniometer.

After the work of Altman and Bland, ${ }^{9}$ scatter plots were constructed (-Figs. $\mathbf{3}$ and $\mathbf{4}$ ) which demonstrate linearity, proportionality, and a close approximation to the "ideal" $\mathrm{x}=\mathrm{y}$ line. The standard deviation of the difference and Pearson's product-moment correlations were calculated with $95 \%$ confidence intervals ( - Table $\mathbf{1}$ ).

\section{Results}

For intraobserver reliability the Lafayette goniometer had a SD of differences of 4.8 degrees. Statistically, therefore, $95 \%$ of measurements should lie \pm 9.6 degrees. The interobserver reliability was \pm 8.4 degrees. By comparison the iPhone had an interobserver reliability \pm 2.7 degrees and an intraobserver reliability \pm 4.6 degrees.

Overall the iPhone goniometer achieved better interobserver and intraobserver reliabilities compared with the Lafayette goniometer.

The accuracy (validity) of the iPhone goniometer, compared with the standard Lafayette goniometer was \pm 7.6 degrees with a mean difference of 0.38 degrees (see - Fig. 4).

\section{Discussion}

Loss of knee extension is a well-recognized complication following ACL reconstruction surgery and carries with it poor functional results. Sachs et $\mathrm{al}^{1}$ reviewed 126 patients who had undergone ACL reconstruction. A flexion contracture of 5 degrees or more was present in $24 \%$ of patients and correlated positively with patellofemoral pain. Roe et $\mathrm{al}^{10}$ evaluated patients at 7 years after ACL reconstruction. The authors concluded that a flexion contracture was associated with early osteoarthritic changes. Kocher et $\mathrm{al}^{11}$ evaluated 201 patients and found that patients with a knee extension within 5 degrees of the opposite normal knee had a median patient satisfaction score of 9 (scale 1 [worst] to 10 [best]) compared with a median score of 7 for patients who lacked more than 5 degrees of knee extension. Shelbourne and $\mathrm{Gray}^{2}$ performed a regression analysis on 502 patients following ACL reconstruction at a minimum follow-up of 10 years. They found that the most statistically significant factor relating to lower subjective scores was a lack of normal knee extension. They found that even 3 to 5 degrees loss of knee extension compared with the opposite knee adversely affected subjective (International Knee Documentation Committee and Noyes subjective knee scores) and objective (isokinetic quadriceps muscle strength) outcomes.

Measurement of subtle loss of knee flexion can be difficult. The anatomy of the femur makes the identification of bony landmarks difficult and, in some patients, virtually impossible. Lenssen et al, ${ }^{12}$ when measuring range of movement in patients knees with a long-arm goniometer found that based on their interobserver agreement, the SDDs for active extension was 8.2 degrees, that is, only changes in motion greater than this value would be detected beyond measurement error when different clinicians performed similar measurements. Bovens et $\mathrm{al}^{4}$ in a study to determine the variability and reliability of joint measurements determined that it is difficult to show an improvement or worsening of a joint motion of less than 5 to 10 degrees. Such errors may exceed the magnitude of a significant fixed flexion deformity. Our results for the Lafayette goniometer are comparable with similar studies of conventional goniometers. ${ }^{1,2}$

Long-leg lateral radiographs of the lower extremity are technically difficult and involve a significant ionizing radiation exposure. Two-dimensional digital image analysis has been described ${ }^{13,14}$ but is dependent on body habitus and specialist input. Bennet et $\mathrm{al}^{13}$ described a method of measuring and recording knee joint motion using digital imaging which demonstrated a high interobserver reliability $(r>0.948)$ and intraobserver repeatability $(r>0.906)$. In a busy clinic setting, recording and measuring digital photographs are time-consuming and impractical. Volumetric imaging with computed tomography or magnetic resonance imaging remains time consuming and expensive. While computer navigation systems, ${ }^{15}$ are increasingly being used in the operating theater, they are seldom available in the outpatient setting. Furthermore, no commonly used method can be used by the patient himself or herself to assess his or her own progress.

The speed and ease of use of a smartphone-based goniometer, coupled to the convenience of a device that is always to 

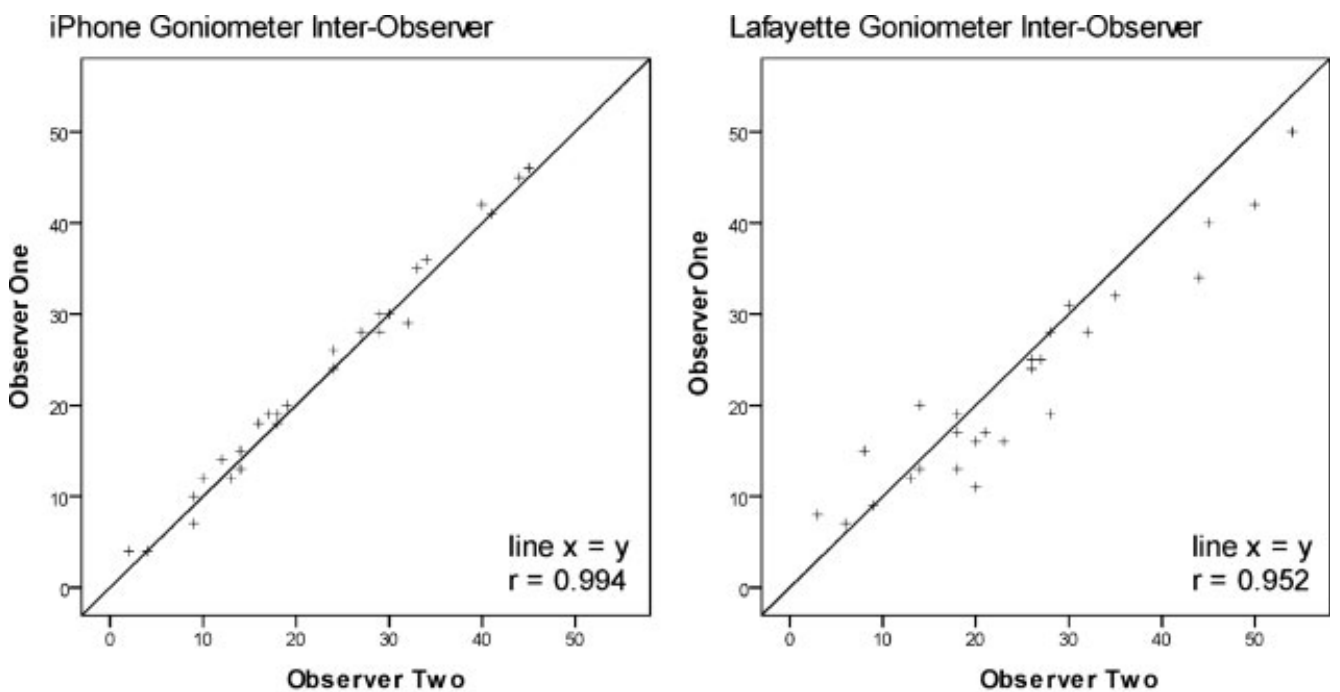

iPhone Goniometer Intra-Obsener

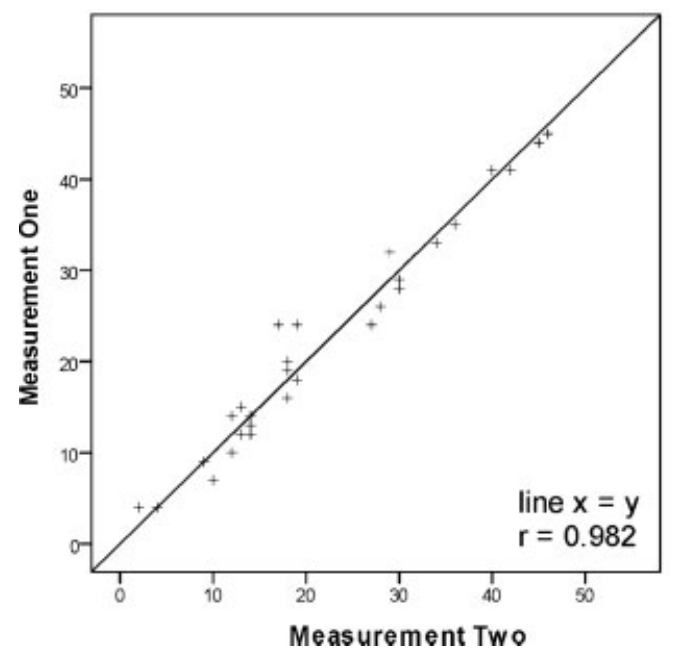

\section{Lafayette Goniometer Intra-Observer}

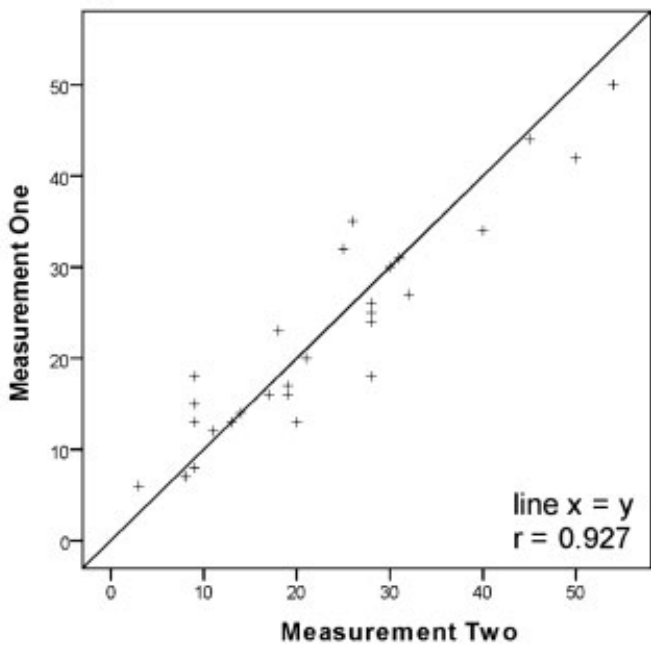

Figure 3 Scatter plots demonstrating interobserver and intraobserver correlation for the Lafayette goniometer and the iPhone goniometer.

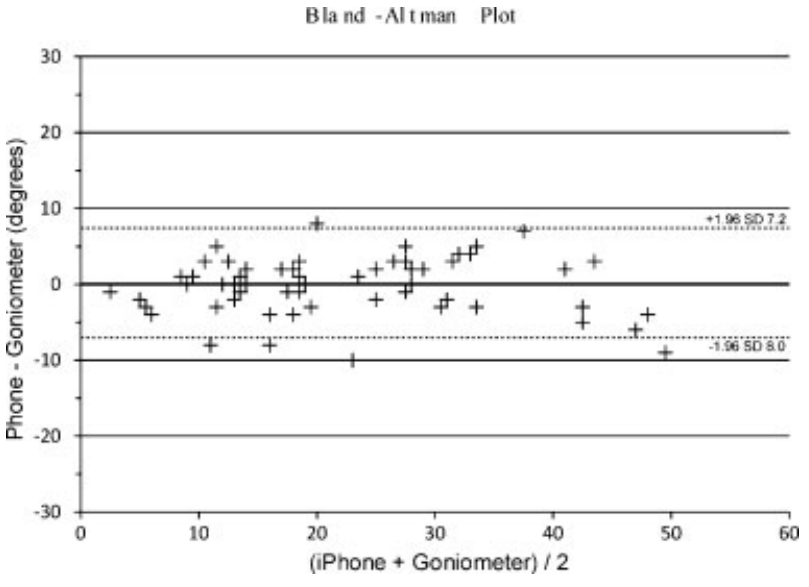

Figure 4 Bland and Altman plot ${ }^{9}$ of measurement differences against average readings by the Lafayette and iPhone goniometers. 95\% confidence interval is shown. This plot shows the spread of agreement between the measuring techniques, the closer the mean difference lies to zero, the more proportional the two techniques being compared. The spread of the data, represented by the $95 \% \mathrm{Cl}$ (1.96 SD) lines demonstrates the reliability with which the two techniques agree (a narrower range is better). hand, make it a potentially useful tool provided that it is both valid and reliable.

In our study, we found the iPhone goniometer to have excellent reliability. It compared favorably with the Lafayette goniometer, having an interobserver correlation (r) of 0.994 compared with 0.952 for the Lafayette. It also exhibited better intraobserver correlation ( $\mathrm{r}=0.982$ compared with 0.927 ). The iPhone instrument also had excellent validity when compared with the Lafayette goniometer. The datasets from the two instruments correlate closely $(r=0.947)$ are proportional and have mean difference of only -0.4 degrees (SD 3.86 degrees).

From our data, we calculate the SDD measureable by iPhone goniometry to be better than half that measureable with a telescopic-armed goniometer.

In conclusion, we found the iPhone goniometer to be a reliable tool for the measurement of subtle knee flexion in the clinic setting when compared with the current standard bedside technique. While comparison against radiographic studies would be of value we feel that the radiological exposure would not be justified in routine 
Table 1 Summary of the Interobserver and Intraobserver Reliability of the Two Measurement Techniques and Assessment of the Validity of the iPhone Goniometer Compared with the "Standard" Lafayette Goniometer (after Bland et al)

\begin{tabular}{|l|l|l|l|l|}
\hline & $\begin{array}{l}\text { Pearson's } \\
\text { Correlation ( } r)\end{array}$ & $\begin{array}{l}\text { 95\% Confidence } \\
\text { Interval }\end{array}$ & $\begin{array}{l}\text { Mean } \\
\text { Differences }\end{array}$ & $\begin{array}{l}\text { SD of Differences } \\
\text { (Degrees) }\end{array}$ \\
\hline Lafayette goniometer & 0.927 & $(0.851,0.965)$ & 0.7 & 4.80 \\
\hline Intraobserver & & & & \\
\hline Lafayette goniometer & 0.952 & $(0.901,0.977)$ & 2.0 & 4.18 \\
\hline Interobserver & & & & \\
\hline iPhone & 0.982 & $(0.962,0.991)$ & 0.0 & 2.30 \\
\hline Intraobserver & & & & \multicolumn{2}{|l|}{} \\
\hline iPhone & 0.994 & $(0.986,0.997)$ & -0.7 & 1.37 \\
\hline Interobserver & & & \multicolumn{2}{|l|}{} \\
\hline Lafayette goniometer vs. iPhone & 0.947 & $(0.913,0.968)$ & 0.4 & 3.86 \\
\hline
\end{tabular}

clinical practice and could not, therefore, be sanctioned in this context.

\section{References}

1 Sachs RA, Daniel DM, Stone ML, Garfein RF. Patellofemoral problems after anterior cruciate ligament reconstruction. Am J Sports Med 1989;17(6):760-765

2 Shelbourne KD, Gray T. Minimum 10-year results after anterior cruciate ligament reconstruction: how the loss of normal knee motion compounds other factors related to the development of osteoarthritis after surgery. Am J Sports Med 2009;37(3):471480

3 Boone DC, Azen SP, Lin CM, Spence C, Baron C, Lee L. Reliability of goniometric measurements. Phys Ther 1978;58(11):1355-1360

4 Bovens AM, van Baak MA, Vrencken JG, Wijnen JA, Verstappen FT. Variability and reliability of joint measurements. Am J Sports Med 1990;18(1):58-63

5 Edwards JZ, Greene KA, Davis RS, Kovacik MW, Noe DA, Askew MJ. Measuring flexion in knee arthroplasty patients. J Arthroplasty 2004;19(3):369-372

6 Cleffken B, van Breukelen G, Brink P, van Mameren H, Olde Damink S. Digital goniometric measurement of knee joint motion. Evaluation of usefulness for research settings and clinical practice. Knee 2007;14(5):385-389
7 Anderson M, Messner MB, Green WT. Distribution of lengths of the normal femur and tibia in children from one to eighteen years of age. J Bone Joint Surg Am 1964;46:1197-1202

8 Ockendon M. Available at: http://www.ockendon.net. 2010

9 Bland JM, Altman DG. Statistical methods for assessing agreement between two methods of clinical measurement. Lancet 1986;1 (8476):307-310

10 Roe J, Pinczewski LA, Russell VJ, Salmon LJ, Kawamata T, Chew M. A 7-year follow-up of patellar tendon and hamstring tendon grafts for arthroscopic anterior cruciate ligament reconstruction: differences and similarities. Am J Sports Med 2005;33(9):1337-1345

11 Kocher MS, Steadman JR, Briggs K, Zurakowski D, Sterett WI, Hawkins RJ. Determinants of patient satisfaction with outcome after anterior cruciate ligament reconstruction. J Bone Joint Surg Am 2002;84-A(9):1560-1572

12 Lenssen AF, van Dam EM, Crijns YH, et al. Reproducibility of goniometric measurement of the knee in the in-hospital phase following total knee arthroplasty. BMC Musculoskelet Disord 2007;8:83

13 Bennett D, Hanratty B, Thompson N, Beverland D. Measurement of knee joint motion using digital imaging. Int Orthop 2009;33(6): $1627-1631$

14 Russell TG, Jull GA, Wootton R. Can the Internet be used as a medium to evaluate knee angle? Man Ther 2003;8(4):242-246

15 Austin MS, Ghanem E, Joshi A, Trappler R, Parvizi J, Hozack WJ. The assessment of intraoperative prosthetic knee range of motion using two methods. J Arthroplasty 2008;23(4):515-521 